\title{
Rough Sets and Algebras of Relations
}

\author{
Ivo Düntsch \\ School of Information and Software Engineering \\ University of Ulster at Jordanstown \\ Newtownabbey, BT 37 0QB, N.Ireland \\ I.Duentsch@ulst.ac.uk
}

February 5, 1999

\begin{abstract}
A survey of results is presented on relationships between the algebraic systems derived from the approximation spaces induced by information systems and various classes of algebras of relations. Rough relation algebras are presented and it is shown that they form a discriminator variety. A characterisation of the class of representable rough relation algebras is given. The family of closure operators derived from an approximation space is abstractly characterised as certain type of Boolean algebra with operators. A representation theorem is given which says that every such an algebra is isomorphic with a similar algebra that is derived from an information system.
\end{abstract}

\section{Notation and definitions}

The history and the impact of rough sets as a means of modelling incomplete information are considered elsewhere in this Volume, so we shall be content to just state the basic notions. Let $U$ be a set and $\theta$ an equivalence relation on $U$. The pair $\langle U, \theta\rangle$ will be called an approximation space. For $A \subseteq U$, $A_{u}=\bigcup\{\theta x: x \in A\}$ is the upper approximation of $A$, and $A \subseteq U, A_{d}=\bigcup\{\theta x: \theta x \subseteq A\}$ is its lower approximation. A rough subset of $U$ with respect to $\theta$ is a pair $\left\langle X_{d}, X_{u}\right\rangle$ with $X \subseteq U$. The collection of all rough subsets of $U$ with respect to $\theta$ is denoted by $S b_{r}^{\theta}(U)$, and will sometimes be called a full algebra of rough sets. If $\theta$ is understood, we shall just write $S b_{r}(U)$

\section{Rough Sets and Regular Double Stone Algebras}

An algebraic approach to rough sets was first proposed in (Iw1). Iwinski's aim - later extended by (PP1) - was to endow the rough subsets of $U$ with a natural algebraic structure. It turns out that regular double Stone algebras are the proper setting.

A double Stone algebra (DSA) $\left\langle L,+, \cdot,{ }^{*},{ }^{+}, 0,1\right\rangle$ is an algebra of type $\langle 2,2,1$, $1,0,0\rangle$ such that

1. $\langle L,+, \cdot, 0,1\rangle$ is a bounded distributive lattice, 
2. $x^{*}$ is the pseudocomplement of $x$, i.e. $y \leq x^{*} \Leftrightarrow y \cdot x=0$,

3. $x^{+}$is the dual pseudocomplement of $x$, i.e. $y \geq x^{+} \Leftrightarrow y+x=1$,

4. $x^{*}+x^{* *}=1, x^{+} \cdot x^{++}=0$.

Conditions 2. and 3. are equivalent to the equations

$$
\begin{array}{ll}
x \cdot(x \cdot y)^{*}=x \cdot y^{*}, & x+(x+y)^{+}=x+y^{+} \\
x \cdot 0^{*}=x, & x+1^{+}=x \\
0^{* *}=0, & 1^{++}=1
\end{array}
$$

so that DSA is an equational class. $\mathrm{L}$ is called regular, if it additionally satisfies the equation $x \cdot x^{+} \leq$ $y+y^{*}$. This is equivalent to $x^{+}=y^{+}$and $x^{*}=y^{*}$ imply $x=y$.

The center $B(L)=\left\{x^{*}: x \in L\right\}$ of $L$ is a subalgebra of $L$ and a Boolean algebra, in which * and + coincide with the Boolean complement which we denote by -. An element of the centre of $L$ will also be called a Boolean element. The dense set $\left\{x \in L: x^{*}=0\right\}$ of $L$ is denoted by $D(L)$, or simply $D$, if $L$ is understood. For any $M \subseteq L, M^{+}$is the set $\left\{x^{+}: x \in M\right\}$.

A construction of regular double Stone algebras which is important for our purposes is given by

Lemma 2.1. (Kal) Let $\langle B,+, \cdot,-, 0,1\rangle$ be a Boolean algebra and $F$ be a not necessarily proper filter on B. Set

$$
\langle B, F\rangle=\{\langle a, b\rangle \in B \times B: a \leq b \text { and }-b+a \in F\} .
$$

Then, $L=\langle B, F\rangle$ is a 0,1 - sublattice of $B \times B$, and it becomes a regular double Stone algebra by setting

$$
\langle a, b\rangle^{*}=\langle-b,-b\rangle,\langle a, b\rangle^{+}=\langle-a,-a\rangle
$$

Furthermore, $B(L) \cong B$ as Boolean algebras, and $D(L) \cong F$ as lattices. Note that

$$
B(L)=\{\langle a, a\rangle: a \in B\}, D(L)=\{\langle a, 1\rangle: a \in F\} .
$$

Conversely, if $M$ is a regular double Stone algebra, $B=B(M), F=D(M)^{++}$, then the mapping which assigns to each $x \in M$ the pair $\left\langle x^{++}, x^{* *}\right\rangle$ is an isomorphism between $M$ and $\langle B, F\rangle$.

If $F=B$, then $\langle B, F\rangle$ is also denoted by $B^{[2]}$.

In view of things to come it is useful to note that by Lemma 2.1 each element $x$ of a regular double Stone algebra is uniquely described by the greatest Boolean element below $x$ and the smallest Boolean element above $x$.

Now, suppose that $\langle U, \theta\rangle$ is an approximation space. We can view the classes of $\theta$ as atoms of a complete subalgebra of the Boolean algebra $S b(U)$. Conversely, any atomic complete subalgebra $B$ of $S b(U)$ gives rise to an equivalence relation $\theta$ on $U$, and this correspondence is bijective. The elements of $B$ are $\emptyset$ and the unions of classes of its associated equivalence relation. If $\{a\} \in B$, then, for every $X \subseteq U$ we have 
If $a \in X_{u}$, then $a \in X$, and the rough sets of the corresponding approximation space are the elements of the regular double Stone algebra $\langle B, F\rangle$, where $F$ is the filter of $B$ which is generated by the union of singleton elements of $B^{1}$. Note that, if $\theta$ is the identity on $U$, then $F=\{U\}$, and we see that the full algebra of rough sets on $U$ need not be of the form $B^{[2]}$. At any rate, we have

Proposition 2.2. (Iw $1, P P 1, C o 2)$ Suppose that $\langle U, \theta\rangle$ is an approximation space. Then, $S b_{r}^{\theta}(U)$ is a regular double Stone algebra with the operations of Lemma 2.1.

Steve Comer has shown that a converse also holds:

Proposition 2.3. (Co1, Co2) Let L be a regular double Stone algebra. Then, there is an approximation space $\langle U, \theta\rangle$ such that $L$ is isomorphic to a subalgebra of $S b(U)^{[2]}$

Proof. Every Stone algebra can be embedded into an algebra of the form $B^{[2]}$, where $B$ is a complete and atomic Boolean algebra, see (BG1). As remarked above, each such algebra is isomorphic to a full algebra of rough sets.

\section{Rough Relation Algebras}

Pawlak's original approach to model incomplete information was to take sets as the basic entity. However, sets can themselves have an underlying structure or a special form. Subsequently, (Co2) proposed to look at the case where the underlying sets are binary relations, and how incomplete information about these can be modelled.

If we are given a set $U$, then the subsets of $U$ can be thought of as truth sets of unary predicates, and appropriate operations on subsets of $U$ are the usual Boolean ones which, in the case of rough sets, lead to certain regular double Stone algebras. If we look at binary relations we have additional natural operations, namely, relational composition $\circ$, relational converse ${ }^{-1}$, and the identity relation $1^{\prime}$ as a new constant: Here,

$$
\begin{aligned}
R \circ S & =\{\langle a, c\rangle \in U \times U: \text { There is some } b \in U \text { such that } R(a, b) \text { and } S(b, c)\}, \\
R^{-1} & =\{\langle b, a\rangle: R(a, b)\}, \\
1^{\prime} & =\{\langle a, a\rangle: a \in U\} .
\end{aligned}
$$

With these operations we can view the set of all binary relations on $U$ as an algebra

$$
\operatorname{Rel}(U)=\left\langle\operatorname{Sb}\left(U^{2}\right), \cup, \cap,-, \emptyset, U \times U,^{*},{ }^{-1}, 1^{\prime}\right\rangle,
$$

called the full algebra of binary relations on $U$. Any subalgebra of $\operatorname{Rel}(U)$ is called an algebra of binary relations $(B R A)$ on $U$.

Tarski has introduced a class of algebras which generalizes the notion of BRA:

A relation algebra (RA) $\left\langle A,+, \cdot,-, 0,1, \circ,{ }^{-1}, 1^{\prime}\right\rangle$ is a structure of type $\langle 2,2,1$, $0,0,2,1,0\rangle$ which satisfies

\footnotetext{
${ }^{1}$ I should like to thank Piero Pagliani for pointing this out.
} 
1. $\langle A,+, \cdot,-, 0,1\rangle$ is a Boolean algebra.

2. $\left\langle A, \circ,{ }^{-1}, 1^{\prime}\right\rangle$ is an involuted monoid.

3. For all $a, b, c$ the following conditions are equivalent:

$$
(a \circ b) \cdot c=0,\left(a^{-1} \circ c\right) \cdot b=0,\left(c \circ b^{-1}\right) \cdot a=0 .
$$

It can be shown that the class of RAs is equational. For the background and the relevant facts of RAs the reader is invited to consult ( $\mathrm{Jo} 2)$ or (TG1).

A relation algebra $A$ is called representable, if it is a subalgebra of a product of full algebras of binary relations.

Now, we shall generalize RAs to rough structures (Co2): Let $\langle U, \theta\rangle$ be an approximation space, and set $V=U \times U$. $\theta$ defines in a canonical way an equivalence ${ }^{2} \theta$ on $V$ by

$$
\langle x, y\rangle \equiv_{2}\langle u, v\rangle \text { iff } x \equiv_{\theta} u \text { and } y \equiv_{\theta} v
$$

and $\left\langle V,{ }^{2} \theta\right\rangle$ is an approximation space.

A rough relation on $\langle U, \theta\rangle$ is a rough subset of $\left\langle V,{ }^{2} \theta\right\rangle$. In other words, a rough relation on $\langle U, \theta\rangle$ is a pair $\left\langle S_{d}, S_{u}\right\rangle$, where $S \subseteq V$ and for all $\langle a, b\rangle \in V$

$$
\begin{array}{ll}
\langle a, b\rangle \in S_{d} \quad \Leftrightarrow^{2} \quad \theta\langle a, b\rangle \subseteq S, \\
\langle a, b\rangle \in S_{u} \quad \Leftrightarrow^{2} \quad \theta\langle a, b\rangle \cap S \neq \emptyset .
\end{array}
$$

(Recall that ${ }^{2} \theta\langle a, b\rangle$ is the equivalence class of $\langle a, b\rangle$ with respect to ${ }^{2} \theta$.)

$S b_{r}^{2} \theta(V)$ is a regular double Stone algebra by the results of the preceding section. We define the additional relational operators on $S b_{r}^{2} \theta(V)$ as follows:

$$
\begin{aligned}
\left\langle T_{d}, T_{u}\right\rangle \circ\left\langle S_{d}, S_{u}\right\rangle & =\left\langle T_{d} \circ S_{d}, T_{u} \circ S_{u}\right\rangle \\
\left\langle S_{d}, S_{u}\right\rangle^{-1} & =\left\langle S_{d}^{-1}, S_{u}^{-1}\right\rangle \\
1^{\prime} & =\langle\theta, \theta\rangle .
\end{aligned}
$$

The structure $\left\langle S b_{r}^{2} \theta(V), \cup, \cap,{ }^{*},+, \emptyset, V, \circ,{ }^{-1},\langle\theta, \theta\rangle\right\rangle$ is called the full algebra of rough relations over $\langle U, \theta\rangle$. Subalgebras of $S b_{r}^{2} \theta(V)$ are called algebras of rough relations.

Rough relation algebras are a generalization of relation algebras, where the Boolean part is replaced by a regular double Stone algebra, and the following set of axioms was proposed by (Co2):

A rough relation algebra $\left(\mathrm{R}^{2} \mathrm{~A}\right)$ is an algebra

$$
\left\langle L,+, \cdot,^{*},{ }^{+}, 0,1, ;,{ }^{-1}, 1^{\prime}\right\rangle
$$

such that

(i). $\left\langle L,+, \cdot,^{*},{ }^{+}, 0,1\right\rangle$ is a regular double Stone algebra,

(ii). $(x ; y) ; z=x ;(y ; z)$, 
(iii). $(x+y) ; z=x ; z+y ; z$,

(iv). $x ; 1^{\prime}=1^{\prime} ; x=x$,

(v). $\left(x^{-1}\right)^{-1}=x$,

(vi). $(x+y)^{-1}=x^{-1}+y^{-1}$,

(vii). $(x ; y)^{-1}=y^{-1} ; x^{-1}$,

(viii). $\left(x^{-1} ;(x ; y)^{*}\right) \cdot y=0$,

(ix). $(x ; y) \cdot z \leq x ; x^{-1} ; z$,

(x). $\left(x^{*} ; y^{*}\right)^{* *}=x^{*} ; y^{*}$,

(xi). $\left(1^{\prime}\right)^{* *}=1^{\prime}$.

If $B$ is a relation algebra, then $B^{[2]}$ becomes a rough relation algebra if ; and ${ }^{-1}$ are defined componentwise. We shall denote this algebra by $B_{r}^{[2]}$.

It is clear that every full algebra of rough relations is an $\mathrm{R}^{2} \mathrm{~A}$. A rough relation algebra is representable, if it is a subalgebra of a product of full algebras of rough relations.

Many equations which hold in RAs also hold in $\mathrm{R}^{2}$ As. Some properties specific to rough relation algebras are given in

Proposition 3.1. (Co2, Dul) Let L be an $R^{2} A$. Then,

1. $B(L)$ is closed under; and ${ }^{-1}$, and $1^{\prime} \in B(L)$.

2. $B(L)$ is a relation algebra and a subalgebra of $L$.

3. If $L \cong S b_{r}^{2} \theta(V)$ for some approximation space, then $B(L) \cong \operatorname{Rel}(U / \theta)$.

4. $D(L)$ is closed under; and ${ }^{-1}$.

5. $(x ; y)^{* *}=x^{* *} ; y^{* *}$ for all $x, y \in L$.

Proof. 1. The closure of $B(L)$ under ; and $1^{\prime}$ are just axioms (x) and (xi). Since ${ }^{-1}$ distributes over *, and $\left(a^{-1}\right)^{-1}=a$, we have

$$
a^{*} \cdot a=0 \Rightarrow\left(a^{*}\right)^{-1} \cdot a^{-1}=0 \Rightarrow\left(a^{*}\right)^{-1} \leq\left(a^{-1}\right)^{*},
$$

and conversely,

$$
a^{-1} \cdot\left(a^{-1}\right)^{*}=0 \Rightarrow a \cdot\left(\left(a^{-1}\right)^{*}\right)^{-1}=0 \Rightarrow\left(\left(a^{-1}\right)^{*}\right)^{-1} \leq a^{*} \Rightarrow\left(a^{-1}\right)^{*} \leq\left(a^{*}\right)^{-1} .
$$

2. To show that L satisfies condition 3. for RAs, one can use 2.1. of (CT1) which goes through unchanged. Clearly, $B(L)$ is a subalgebra of $L$.

3. This follows immediately from the definition of $B(L)$. 
4. Let $x, y \in D(L)$. Then,

$$
1=x^{* *}=y^{* *}=x^{* *} ; y^{* *}=(x ; y)^{* *},
$$

and therefore $(x ; y)^{*}=0$.

5. Clearly, $x \leq x^{* *}$ and $y \leq y^{* *}$ imply

$$
(x ; y)^{* *} \leq\left(x^{* *} ; y^{* *}\right)^{* *}=x^{* *} ; y^{* *}
$$

For the converse assume that $(x ; y)^{* *} \lessgtr x^{* *} ; y^{* *}$. Then, $\left(x^{* *} ; y^{* *}\right) \cdot(x ; y)^{*} \geqslant 0$, and

$$
\begin{aligned}
\left(\left(x^{-1}\right)^{* *} ;(x ; y)^{*}\right) \cdot y^{* *} & \gtrless 0, \\
\left(\left(x^{-1}\right)^{* *} ;(x ; y)^{*}\right) \cdot y & \gtrless 0 \\
\left(x^{* *} ; y\right) \cdot(x ; y)^{*} & \gtrless 0 \\
\left((x ; y)^{*} \cdot y^{-1}\right) \cdot x^{* *} & \gtrless 0 \\
\left((x ; y)^{*} \cdot y^{-1}\right) \cdot x & \gtrless 0 \\
(x ; y) \cdot(x ; y)^{*} & \gtrless 0,
\end{aligned}
$$

a contradiction.

Just like relation algebras, $\mathrm{R}^{2}$ As have very strong structural properties: An algebra $A$ is a discriminator algebra if there is some term operation $f$ in the language of $A$ such that

$$
f(a, b, c)= \begin{cases}c, & \text { if } a=b, \\ a, & \text { otherwise }\end{cases}
$$

A variety $\mathbf{V}$ is called a discriminator variety if it is generated by a class $K$ of algebras such that some term operation $f$ in the language of $\mathbf{V}$ represents the discriminator term as above on each member of $K$. Discriminator algebras have, among others, the following pleasant properties, see (JAN1):

Proposition 3.2. Let $\mathbf{V}$ be a discriminator variety. Then,

1. $\mathbf{V}$ is congruence permutable, congruence distributive, congruence extensile, and semisimple.

2. For every non trivial algebra $A$ in $\mathbf{V}$ the following are equivalent:

(a) A is simple.

(b) A is subdirectly irreducible.

(c) A is directly indecomposable.

3. There is an effective way of associating with each open Horn formula $\varphi$ in the language of $\mathbf{V}$ an equation $\sigma_{\varphi}$ in this language such that $\varphi$ and $\sigma_{\varphi}$ have the same truth set in every simple member of $\mathbf{V}$. 
To show that $\mathrm{R}^{2} \mathrm{~A}$ is a discriminator variety we first state

Lemma 3.3. (Dul) Let $L$ be an $R^{2} A$ and $\psi \in C o n(B(L))$. Then,

1. $\psi_{L} \in \operatorname{Con}(L)$.

2. $\operatorname{Con}(L) \cong \operatorname{Con}(B(L))$.

We now have

Proposition 3.4. (Dul) The variety of $R^{2} A s$ is a discriminator variety.

Proof. It is enough to show that a simple $\mathrm{R}^{2} \mathrm{~A} L$ is a discriminator algebra. For $a, b \in B(L)$ we denote the symmetric difference by $a \otimes b$; recall that $a=b \Leftrightarrow a \otimes b=0$. Set

$$
\tau(a, b)=1 ;\left(a^{* *} \otimes b^{* *}+a^{++} \otimes b^{++}\right) ; 1 .
$$

Then,

$$
\tau(a, b)= \begin{cases}1, & \text { if } a \neq b \\ 0, & \text { otherwise }\end{cases}
$$

Let $a=b$; then, since $L$ is regular, $a^{* *}=b^{* *}$ and $a^{++}=b^{++}$, and thus $a^{* *} \otimes b^{* *}=a^{++} \otimes b^{++}=0$. It follows that

$$
\left.\left[1 ;\left(a^{* *} \otimes b^{* *}\right) ; 1\right]+\left[1 ;\left(a^{++} \otimes b^{++}\right) ; 1\right]=1 ;\left[\left(a^{* *} \otimes b^{* *}\right)\right)+\left(a^{++} \otimes b^{++}\right)\right]=\tau(a, b)=0 .
$$

Conversely, let $a \neq b$. Then, $a^{* *} \neq b^{* *}$ or $a^{++} \neq b^{++}$. Since $L$ is simple, so is $B(L)$ by Lemma 3.3. We know from (Jo1) that for a relation algebra $A$ and $x \in A$,

$$
x \neq 0 \Leftrightarrow 1 ; x ; 1=1 .
$$

Therefore,

$$
1 ;\left(a^{* *} \otimes b^{* *}\right) ; 1=1 \text { or } 1 ;\left(a^{++} * b^{++}\right) ; 1=1,
$$

since $a^{* *}, b^{* *}, a^{++}, b^{++}$are Boolean. Consequently,

$$
1=\left[1 ;\left(a^{* *} \otimes b^{* *}\right) ; 1\right]+\left[1 ;\left(a^{++} \otimes b^{++}\right) ; 1\right]=\tau(a, b) .
$$

Now, set

$$
\sigma(a, b, c)=\tau(a, b) \cdot a+\tau(a, b)^{*} \cdot c .
$$

If $a=b$, then $\tau(a, b)=0$, and hence $\sigma(a, b, c)=\tau(a, b)^{*} \cdot c=c$. If $a \neq b$, then $\tau(a, b)=1$, and therefore $\sigma(a, b, c)=\tau(a, b) \cdot a=a$.

Finally, the representable $\mathrm{R}^{2} \mathrm{As}$ can be characterized as follows: 
Proposition 3.5. (Dul) Suppose that $L=\langle B, F\rangle$ is an $R^{2} A$. Then, $L$ is representable if and only if $B(L)$ is a representable relation algebra and $L$ satisfies the equation $(x ; y)^{++}=x^{++} ; y^{++}$.

Proof. Call an $\mathrm{R}^{2} \mathrm{~A}$ canonical which satisfies the equation. To show the result, we first prove some auxiliary results which seem interesting in their own right. Our first claim explains why we call these algebras canonical:

Claim 1. (1) $L$ is canonical if and only if

$$
\langle a, b\rangle ;\langle c, d\rangle=\langle a ; c, b ; d\rangle
$$

for all $\langle a, b\rangle,\langle c, d\rangle \in\langle B, F\rangle$.

Proof. " $\Rightarrow$ ": Suppose $L$ is canonical, and let $\langle a, b\rangle,\langle c, d\rangle \in\langle B, F\rangle$. Then, there are $x, y \in L$ such that $a=x^{++}, b=x^{* *}, c=y^{++}, d=y^{* *}$, and

$$
\begin{aligned}
\langle x++, x * *\rangle ;\langle y++, y * *\rangle & =x ; y \\
& =\langle(x ; y)++,(x ; y) * *\rangle \\
& =\langle x++; y++, x * * ; y * *\rangle .
\end{aligned}
$$

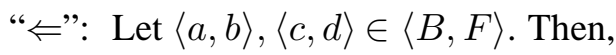

$$
(\langle a, b\rangle ;\langle c, d\rangle)^{++}=\langle a ; c, b ; d\rangle^{++}=\langle a ; c, a ; c\rangle=\langle a, a\rangle ;\langle c, c\rangle=\langle a, b\rangle^{++} ;\langle c, d\rangle^{++},
$$

which proves the claim.

Claim 2. (2) For any $L, B(L)_{r}^{[2]}$ is canonical and, if $L$ is canonical, then it is a subalgebra of $B(L)_{r}^{[2]}$.

Proof. This follows immediately from the definition of $B_{r}^{[2]}$ and Claim 1.

Claim 3. (3) If $C$ is (isomorphic to) a full relation algebra $\operatorname{Rel}(U)$, then $C_{r}^{[2]}$ is isomorphic to a full algebra of rough relations.

Proof. Let $U^{\prime}=\left\{x^{\prime}: x \in U\right\}$ be disjoint from $U$, and set $V=U \cup U^{\prime}$. Define an equivalence relation $\theta$ on $V$ by identifying $x$ and $x^{\prime}$, so that $\theta x=\left\{x, x^{\prime}\right\}$. Then, $B\left(R_{V}\right)$ and $C$ are isomorphic as relation algebras. Let $\left\langle R_{d}, R_{d}\right\rangle \in B\left(R_{V}\right)$, and define

$$
S=R \cup\left\{\langle x, y\rangle: x, y \in U, \theta x \times \theta y \subseteq-R_{d}\right\} .
$$

Then, $S_{d}=R_{d}, S_{u}=1$, and thus $\left\langle R_{d}, 1\right\rangle \in D\left(R_{V}\right)$ for each $R \in C$. Now, the mapping defined by $\left\langle R_{d}, R_{d}\right\rangle \mapsto\left\langle R_{d}, 1\right\rangle$ is a lattice isomorphism, and hence $B\left(R_{V}\right) \cong D\left(R_{V}\right)$. It follows that $R_{V}=B\left(R_{V}\right)_{r}^{[2]} \cong C_{r}^{[2]}$.

Now we can prove the Proposition:

" $\Rightarrow$ ": It is enough to show that every full algebra $R_{U}$ of rough relations is canonical. Let $L=R_{U}$ be the full algebra of rough relations over $\langle U, \theta\rangle$. Then, $B(L) \cong \operatorname{Rel}(U / \theta)$, and thus $B(L)$ is a representable relation algebra. 
Next, let $\left\langle R_{d}, R_{u}\right\rangle,\left\langle S_{d}, S_{u}\right\rangle \in R_{U}$. Then,

$$
\begin{aligned}
\left(\left\langle R_{d}, R_{u}\right\rangle,\left\langle S_{d}, S_{u}\right\rangle\right)^{++} & =\left(\left\langle R_{d} ; S_{d}, R_{u}, S_{u}\right\rangle\right)^{++} \\
& =\left\langle R_{d} ; S_{d}, R_{d}, S_{d}\right\rangle \\
& =\left\langle R_{d} ; R_{d}\right\rangle ;\left\langle S_{d}, S_{d}\right\rangle \\
& =\left\langle R_{d} ; R_{u}\right\rangle^{++} ;\left\langle S_{d}, S_{u}\right\rangle^{++}
\end{aligned}
$$

" $\Leftarrow$ ": Suppose that $L=\langle B, F\rangle$ is canonical, and that $B$ is a representable relation algebra. Since $L \leq B_{r}^{[2]}$ by Claim 2, we can assume that $L=B_{r}^{[2]}$; furthermore, we may assume by 3.4 and 3.2 that $L$ is simple. Then, $B$ is simple by 3.3 , and, since it is representable, there is some set $U$ such that $B \leq \operatorname{Rel}(U)$. It follows that $L=B_{r}^{[2]} \leq \operatorname{Rel}(U)_{r}^{[2]}$, which is representable by Claim 3 .

In particular, the representable rough relation algebras form an equational class.

Relation algebras are a special case of the more general concept of Boolean algebras with operators. Motivated by rough relation algebras, Steve (Co3) has investigated the theory of regular double Stone algebras with operators.

\section{Information Systems}

In the previous sections we have looked at the algebraic structure arising from one given approximation space, and we have considered the special case when the underlying carrier set was the universal binary relation on some other set. In this section we shall describe the algebraic structure of a set of approximation spaces derived from an information system. It turns out that there is a close connection between the resulting structures and cylindric algebras and their derivatives. The standard reference for cylindric algebras are the books (HMT1, HMT2) and we shall refer to these for definitions and results on these algebras. All results in this section are due to Steve Comer.

An information system $S=\langle U, \Omega, V, f\rangle$ as discussed e.g. in (Pa1, Pa2), consists of

1. A set $U$ of objects,

2. A finite set $\Omega$ of attributes,

3. A set $V$ of attribute values,

4. An information function $f: U \times \Omega \rightarrow V$.

We think of $f(u, x)$ as the value which object $u$ takes at the attribute $x$. With each $Q \subseteq \Omega$ we can associate an equivalence relation $\theta_{Q}$ on $U$ by setting

$$
a \equiv_{\theta_{Q}} b \stackrel{\text { def }}{\Longleftrightarrow} f(a, x)=f(b, x) \text { for all } x \in Q
$$

so that $\left\langle U, \theta_{Q}\right\rangle$ is an approximation space.

Intuitively, $a \equiv_{\theta_{Q}} b$ if the objects $a$ and $b$ are indiscernible with respect to the values of their attributes from $Q$. Given $A \subseteq U$, we denote its upper approximation with respect to $\theta_{Q}$ by $\bar{Q} A$, and its lower 
approximation by $\underline{Q} A$. Clearly, $\bar{Q}$ is a closure operator on $\langle S b(U), \subseteq\rangle$, and $\underline{Q}$ is an interior operator. A set $A \subseteq U$ is called definable with knowledge $Q \subseteq \Omega$, if $A$ is a union of equivalence classes of $\theta_{Q}$; equivalently, $A$ is definable from $Q$, iff $\bar{Q} A=\underline{Q} A$.

As an example - which uses rough relations - let us consider the following scenario: Suppose that $U$ is a set of car brands, $\Omega$ a set of attributes associated with cars, e.g. colour, price, reliability etc, $V$ a set of appropriate attributes, and $f$ an information function. Let $R$ be a binary relation on $U$ which was obtained by presenting to a subject two car models, and asking her to decide which she likes better. In order to find out what were the decisive factors in her choice, we can now use rough set methods: Let, as a simple example, $\theta$ be the equivalence on $U$ which identifies cars by their colour. If ${ }^{\theta}\langle a, b\rangle \in R$ and, say, $a$ is red and $b$ is green, we can infer that there is some evidence that she generally prefers green cars over red ones, and if $R={ }^{2}$ \{colour $\} R$, then she is never inconsistent in her choices with respect to colour.

The knowledge approximation algebra $\mathfrak{B}_{S}$ associated with $S$ is the structure

$$
\langle S b(U), \cup, \cap,-, \emptyset, U, \bar{Q}\rangle_{Q \subseteq \Omega}
$$

We note that $\mathfrak{B}_{S}$ is a complete and atomic Boolean algebra with the additional closure operators $\bar{Q}, Q \subseteq \Omega$. If $Q \subseteq \Omega$, the reduct $\langle S b(U), \cup, \cap,-, \emptyset, U, \bar{Q}\rangle$ of $\mathfrak{B}_{S}$ is denoted by $R d_{Q} \mathfrak{B}_{S}$, and it is called an approximation closure algebra.

(Co1) has proposed the following axioms for a class of algebras which are intended to capture the knowledge approximation algebras associated with information systems: An algebra $\mathfrak{B}=\left\langle B,+, \cdot,-, 0,1, \kappa_{P}\right\rangle_{P \subseteq \Omega}$ is a knowledge approximation algebra of type $\Omega$ - called a $\mathrm{KA}_{\Omega}$ - if each $\kappa_{P}$ is a unary operator on $B$, and

A1. $\langle B,+, \cdot,-, 0,1\rangle$ is a complete atomic Boolean algebra,

A2. $\kappa_{P} 0=0$,

A3. $x \leq \kappa_{P} x$,

A4. $\kappa_{P}\left(x \cdot \kappa_{P} y\right)=\kappa_{P} x \cdot \kappa_{P} y$,

A5. If $x \neq 0$, then $\kappa_{\emptyset} x=1$,

A6. $\kappa_{P \cup Q} x=\kappa_{P} x \cdot \kappa_{Q} x$, if $\mathrm{x}$ is an atom of $\mathrm{B}$,

for all $x, y \in B$ and $P, Q \subseteq \Omega$. The class of all knowledge approximation algebras of type $\Omega$ is denoted by $\mathbf{K} \mathbf{A}_{\Omega}$. We note that axioms A1 - A4 tell us that for each $P \subseteq \Omega$, the reduct $R d_{P} \mathfrak{B}_{S}=$ $\left\langle B,+, \cdot,-, 0,1, \kappa_{P}\right\rangle$ of $\mathfrak{B}$ is a cylindric algebra of dimension $1\left(\mathbf{C A}_{1}\right)$ in the sense of (HMT1). Thus, we can regard the operators $\kappa_{P}$ as (in general non - commuting) cylindrifications, and it follows from the corresponding properties of cylindric algebras that the properties given below hold:

Proposition 4.1. (Co1) Let $\mathfrak{B}=\left\langle B,+, \cdot,-, 0,1, \kappa_{P}\right\rangle_{P \subseteq \Omega}$ be an approximation algebra. Then,

1. If $x \leq y$, then $\kappa_{P} x \leq \kappa_{P} y$. 
2. $\kappa_{P} \kappa_{P} x=\kappa_{P} x$.

3. $\sum_{i} \kappa_{P} x_{i}=\kappa_{P}\left(\sum_{i} x_{i}\right)$

4. $\kappa_{P}\left(\prod_{i} \kappa_{P} x_{i}\right)=\prod_{i}\left(\kappa_{P} x_{i}\right)$.

The next result shows that the algebra associated with an information system is a $\mathbf{K} \mathbf{A}_{\Omega}$ :

Proposition 4.2. (Col) Let $S=\langle U, \Omega, V, f\rangle$ be an information system, and $\mathfrak{B}_{S}$ be its associated knowledge approximation algebra. Then,

1. $\mathfrak{B}_{\in} \mathbf{K} \mathbf{A}_{\Omega}$,

2. Each $R d_{Q} \mathfrak{B}_{S}$ is a cylindric algebra of dimension one.

Proof. $1 . \mathfrak{B}_{S}$ is a complete and atomic Boolean algebra, and the operations $\bar{Q}$ are closure operators. This implies A1, A2, and A3. For A4 we need to show that $\bar{Q}(C \cap \bar{Q} D)=\bar{Q} C \cap \bar{Q} D$ for all $C, D \subseteq \Omega$ :

" $\subseteq$ ": Let $x \in \bar{Q}(C \cap \bar{Q} D)$. Then, there is some $y \in C \cap \bar{Q} D$ such that $x \equiv_{\theta_{Q}} y$. Hence, $x \in \bar{Q} C$, and, since $\bar{Q} D$ is a union of $\theta_{Q}$ classes, we also have $x \in \bar{Q} D$.

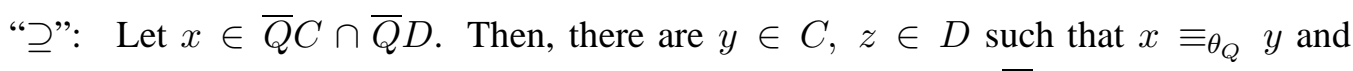
$x \equiv_{\theta_{Q}} z$. Since $D$ is a union of $\theta_{Q}$ classes, we have in fact $y \in \bar{Q} D$, which shows that $x \in \bar{Q}(C \cap \bar{Q} D)$.

A5 follows from the fact that $\theta_{\Omega}={ }^{2} U$. To show A6, let $P, Q \subseteq \Omega$. Because of A5, we can suppose that both $P$ and $Q$ are not empty. Let $x, y \in U$; then,

$$
\begin{aligned}
y \in \overline{P \cup Q}\{x\} & \Leftrightarrow y \equiv_{P \cup Q} x, \\
& \Leftrightarrow f(y, z)=f(x, z) \text { for all } z \in P \cup Q, \\
& \Leftrightarrow \quad f(y, z)=f(x, z) \text { for all } z \in P \\
& \text { and } f(y, z)=f(x, z) \text { for all } z \in Q, \\
& \Leftrightarrow y \theta_{P} x \text { and } y \theta_{Q} x, \\
& \Leftrightarrow y \in \bar{P}\{x\} \cap \bar{Q}\{x\} .
\end{aligned}
$$

2. follows immediately from the definition.

In order to show that the converse also holds, i.e. that the models of the algebras in $\mathbf{K} \mathbf{A}_{\Omega}$ are as expected, we require some preparation. The definition of $\mathbf{K} \mathbf{A}_{\Omega}$ and Proposition 4.1 show that each element of $\mathbf{K A} \mathbf{A}_{\Omega}$ is a completely atomic normal Boolean algebra with operators, i.e. a completely atomic Boolean algebra whose extra operators distribute over arbitrary joins, and do not move 0 .

The completeness of the algebra and the operators imply that each $\kappa_{P}$ is already determined by its values on the atoms of the Boolean part $\mathbf{B}$ of $\mathfrak{B}$. The atomic structure $\mathbf{A t}(\mathbf{B})$ of $\mathfrak{B}$ is $\left\langle A t(B), T_{P}\right\rangle_{P \subseteq \Omega}$, where $A t(B)$ is the set of atoms of $\mathbf{B}$, and for each $P \subseteq \Omega, T_{P}$ is the relation

$$
\left\{\langle x, y\rangle \in A t(B) \times A t(B): y \leq \kappa_{P} x\right\}
$$


Proposition 4.3. (Co1) Let $S$ be an information system and $\mathfrak{B}_{S}$ be its associated $\mathbf{K} \mathbf{A}_{\Omega}$ with atomic structure $\mathbf{A t}\left(\mathbf{B}_{\mathbf{S}}\right)$. Then, for all $P, Q \subseteq \Omega$,

1. $T_{P}$ is an equivalence relation,

2. $T_{\emptyset}={ }^{2} A t\left(B_{S}\right)$,

3. $T_{P} \cap T_{Q}=T_{P \cup Q}$.

In particular, $\mathbf{A t}\left(\mathbf{B}_{\mathbf{S}}\right)$ is a $\cap$ - subsemilattice of the partition lattice $\Pi\left(\operatorname{At}\left(B_{S}\right)\right)$.

Proof. 1. We show that $x T_{P} y \Leftrightarrow \kappa_{P} x=\kappa_{P} y$, from which the claim follows: Let $y \leq \kappa_{P} x$, and assume that $x \not \leq \kappa_{P} y$. Then, since $x$ is an atom of $B$, we have $x \cap \kappa_{P} y=\emptyset$, and A2 and A4 imply that $\kappa_{P} x \cap \kappa_{P} y=\emptyset$. This contradicts $y \leq \kappa_{P} x$. The other direction is obvious.

2. is an immediate consequence of A5.

3. "ᄃ": Let $\langle x, y\rangle \in T_{P} \cap T_{Q}$, i.e. $\kappa_{P} x=\kappa_{P} y$ and $\kappa_{Q} x=\kappa_{Q} y$. Since $x$ and $y$ are atoms and using A4 we obtain

$$
\kappa_{P \cup Q} x=\kappa_{P} x \cap \kappa_{Q} x=\kappa_{P} y \cap \kappa_{Q} y=\kappa_{P \cup Q} y .
$$

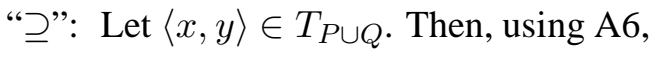

$$
x \leq \kappa_{P \cup Q} x=\kappa_{P \cup Q} y \leq \kappa_{P} y \cap \kappa_{Q} y
$$

shows that $x T_{P} y$ and $x T_{Q} y$.

A relational structure $L=\left\langle U, T_{P}\right\rangle_{P \subseteq \Omega}$ is called a knowledge approximation atom structure, if for all $P, Q \subseteq \Omega$,

1. $T_{P}$ is an equivalence relation on $U$,

2. $T_{\emptyset}={ }^{2} U$,

3. $T_{P} \cap T_{Q}=T_{P \cup Q}$.

If $\Omega$ is finite and not empty, we can associate with each such structure an information system $S(L)$ in the following way: For each $x \in \Omega$ let $V(x)$ be the set of blocks of $T_{\{x\}}$, and set $V=\bigcup_{x \in \Omega}$. Then, define the knowledge function $f: U \times \Omega \rightarrow V$ by

$$
f(u, x)=\text { The block of } T_{\{x\}} \text { containing } u \text {. }
$$

It is easy to see that $S(L)=\langle U, \Omega, V, f\rangle$ is an information system. Furthermore,

Proposition 4.4. (Col) Let $\mathfrak{B}$ be a $K A_{\Omega}, \Omega$ finite, and $L=\mathbf{A t}(\mathbf{B})$ be its atomic structure. Then, $\mathfrak{B} \cong \mathfrak{B}_{S(L)}$. 
Proof. The carrier set $B_{S(L)}$ of $\mathfrak{B}_{S(L)}$ is the power set of $A t(B)$. Thus, the mapping $g: B \rightarrow B_{S(L)}$ defined by $g(b)=\{x \in A t(B): x \leq b\}$ is a Boolean isomorphism.

Now, let $x \in B$, and $P \subseteq \Omega$. We need to show that

$$
g\left(\kappa_{P}^{\mathfrak{B}} x\right)=\kappa_{P}^{\mathfrak{B}_{S(L)}} g(x) .
$$

This is clearly true if $P=\emptyset$, so that we can suppose that $P \neq \emptyset$; indeed, by A6 we may assume that $P$ is an atom of $S b(\Omega)$, say, $P=\{a\}$. Furthermore, by the additivity of $g$ and $\kappa_{P}$, we may suppose that $x$ is an atom of $B$.

Let $y$ be an atom of $B$. Then,

$$
\begin{aligned}
y \in g\left(\kappa_{P}^{\mathfrak{B}} x\right) & \Leftrightarrow y \leq \kappa_{P}^{\mathfrak{B}} x \\
& \Leftrightarrow y T_{P} x \\
& \Leftrightarrow T_{P} y=T_{P} x \\
& \Leftrightarrow f(x, a)=f(y, a) \\
& \Leftrightarrow y \theta_{P} x \\
& \Leftrightarrow y \in \theta_{P} x=\kappa_{P}^{\mathfrak{B}_{S(L)}} x=\kappa_{P}^{\mathfrak{B}_{S(L)}} g(x),
\end{aligned}
$$

and we are done.

This shows that the axioms for knowledge approximation algebras are complete for the intended models. A converse for the second part of 4.2 is given by

Proposition 4.5. (Col)

1. Every complete atomic $\mathbf{C A}_{1}$ is isomorphic to an approximation closure algebra.

2. Every $\mathbf{C A}_{1}$ is embeddable into an approximation closure algebra.

Proof. 1. Let $\left\langle B, c_{0}\right\rangle$ be a complete and atomic $\mathbf{C A}_{1}$, and $\Omega$ be a nonempty finite set. For each non empty $P \subseteq \Omega$ let $\kappa_{P} x=c_{0} x$; also, let $\kappa_{\emptyset} x=0$ if $x=0$, and $\kappa_{\emptyset} x=1$ if $x>0$. Then, $\left\langle B, \kappa_{P}\right\rangle_{P \subseteq \Omega}$ is an approximation algebra, and the rest follows from 4.4

2. By 2.7.20 of (HMT1), each $\mathbf{C A}_{1}$ is embeddable into a complete and atomic one.

The situation regarding the decidability of the first order theory of $\mathbf{K} \mathbf{A}_{\Omega}$ is rather disappointing, though not altogether unexpected:

Proposition 4.6. (Col)

1. If $|\Omega|=1$, then the theory of $\mathbf{K} \mathbf{A}_{\Omega}$ is decidable.

2. If $2 \leq|\Omega|<\omega$ then the theory of $\mathbf{K} \mathbf{A}_{\Omega}$ is undecidable and finitely inseparable.

Proof. 1. Let $|\Omega|=1$. Then, the algebras in $\mathbf{K A}_{\Omega}$ are of the form $\left\langle B, \kappa_{\emptyset}, \kappa_{\Omega}\right\rangle$, where $\left\langle B, \kappa_{\Omega}\right\rangle$ is a completely atomic $\mathbf{C A}_{1}$, and $\kappa_{\Omega}$ is definable in the Boolean part. It was shown in (HMT1) that the theory of complete atomic $\mathbf{C A}_{1}$ 's is the same as the theory of finite $\mathbf{C A}_{1}$ 's, and that it is decidable.

2. Let $\mathbf{E q}$ be the theory of two equivalence relations; it is known that $\mathbf{E q}$ is finitely inseparable, see (Mo1). There it is also shown that to prove that a theory $T$ is finitely inseparable, it is enough to show 
There are formulas $\theta v_{0}, \bar{R} v_{0} v_{1}, \bar{S} v_{0} v_{1}$ in the language of $T$ such that for every finite model $\mathbf{A}=\langle X, R, S\rangle$ of $\mathbf{E q}$ there is a finite model $\mathbf{B}$ of $T$ such that $\left\langle\theta^{\mathbf{B}}, \bar{R}, \overline{\mathbf{B}}, \overline{\mathbf{B}}\right\rangle \cong \mathbf{A}$.

Let $r, s \in \Omega, r \neq s$. We first give a translation of $\mathbf{E q}$ into the language of $\mathbf{K} \mathbf{A}_{\Omega}$ :

$$
\begin{aligned}
\theta v_{0} & : v_{0} \text { is an atom. } \\
\bar{R} v_{0} v_{1} & : \theta v_{0} \wedge \theta v_{1} \wedge \kappa_{\{r\}} v_{0}=\kappa_{\{r\}} v_{1} . \\
\bar{S} v_{0} v_{1} & : \quad \theta v_{0} \wedge \theta v_{1} \wedge \kappa_{\{s\}} v_{0}=\kappa_{\{s\}} v_{1} .
\end{aligned}
$$

If we apply the translation to some $\mathfrak{B} \in \mathbf{K} \mathbf{A}_{\Omega}$, it follows from Proposition 4.3 that $\left\langle A t(B), \bar{R}^{\mathfrak{B}}, \bar{S}^{\mathfrak{B}}\right\rangle$ is a model of Eq.

Finally, let $\mathbf{A}=\langle X, R, S\rangle$ be a finite model of Eq. We obtain a knowledge approximation atom structure $L=\left\langle X, T_{P}\right\rangle_{P \subseteq \Omega}$ by setting

1. $T_{\{r\}}=R, T_{\{s\}}=S, T_{\emptyset}=T_{\{i\}}={ }^{2} X$ for all $i \in \Omega, i \notin\{r, s\}$.

2. $T_{P}=\bigcap\left\{T_{\{i\}}: i \in P\right\}$, for all $P \subseteq \Omega$ with $|P| \geq 2$.

If $\mathbf{A}$ is finite, so is $\mathfrak{B}_{S(L)}$, and it is straightforward to show that $\left\langle A t(B), \bar{R}^{\mathfrak{B}}\right.$, $\left.\bar{S}^{\mathfrak{B}}\right\rangle$ is isomorphic to $\mathbf{A}$.

In (Co2) a close connection of knowledge approximation algebras to a variant of diagonal free cylindric algebras was established. Since a discussion of these results would require an unproportional amount of new definitions and notation, we refer the reader to Comer's paper.

\section{References}

[BG1] Balbes, R. \& Grätzer, G.: Injective and projective Stone algebras. Duke Math. J., 38, (1971), 339-347

[CT1] Chin, L. \& Tarski, A.: Distributive and modular laws in the arithmetic of relation algebras. University of California Publications, 1, (1951), 341-384

[Co1] Comer, S.: An algebraic approach to the approximation of information. Fund. Inform., 14, (1991), 492-502

[Co2] Comer, S.: On connections between information systems, rough sets, and algebraic logic. In: Algebraic Methods in Logic and Computer Science, Banach Center Publications, 28, (1993), 117-124

[Co3] Comer, S.: Perfect extensions of regular double Stone algebras. Algebra Universalis, 34, (1995), 96-109

[Du1] Düntsch, I.: Rough relation algebras. Fund. Inform., 21, (1994), 321-331 
[HMT1] Henkin, L., Monk, J.D. \& Tarski, A.: Cylindric algebras. Part I, North Holland, Amsterdam, (1971)

[HMT2] Henkin, L., Monk, J.D. \& Tarski, A.: Cylindric algebras. Part II, North Holland, Amsterdam, (1985)

[Iw1] Iwinski, T.B.: Algebraic approach to rough sets. Bull. Polish Acad. Sci. Math., 35, (1987), 673-683

[Jo1] Jónsson, B.: Varieties of relation algebras. Algebra Universalis, 15, (1982), 273-298

[Jo2] Jónsson, B.: The theory of binary relations. In: Algebraic Logic, edited by Andréka, H., Monk, J.D. \& Németi, I.: volume 54 of Colloquia Mathematica Societatis János Bolyai, North Holland, Amsterdam, (1991), 245-292

[JAN1] Jónsson, B., Andréka, H. \& Németi, I.: Free algebras in discriminator varieties. Algebra Univ, 28, (1991), 401-447

[Ka1] Katriňák, T.: Construction of regular double $p$-algebras. Bull. Soc. Roy. Sci. Liège, 43, (1974), 294-301

[Mo1] Monk, J.D.: Mathematical logic. Springer, (1976)

[Pa1] Pawlak, Z.: Information systems, theoretical foundations. Information Systems, 6, (1981), 205-218

[Pa2] Pawlak, Z.: Rough sets. Internat. J. Comput. Inform. Sci., 11, (1982), 341-356

[PP1] Pomykala, J. \& Pomykala, J.A.: The Stone algebra of rough sets. Bull. Polish Acad. Sci. Math., 36, (1988), 495-508

[TG1] Tarski, A. \& Givant, S.: A formalization of set theory without variables. Volume 41 of Colloquium Publications, Amer. Math. Soc., Providence, (1987) 